\title{
Conocimientos, Opiniones y Experiencias con la Aplicación de las Políticas de Participación en Salud en Colombia
}

María E. Delgado-Gallego ${ }^{1}$ y María L. Vázquez-Navarrete ${ }^{2}$

${ }^{1}$ Psicóloga. M. Sc. en Psicología Social. Instituto de Psicología de la Universidad del Valle.GEMA. Cali, Colombia. E-mail: mariedel@andinet.com

${ }^{2}$ Médica, Ph. D. en Medicina, M. Sc. in Health Policy, Planning and Financing. Servei d'Estudis del Consorci Hospitalari de Catalunya, Av. Tibidabo 21, 08005, Barcelona, España E-mail: mlvazquez@chc.es

Recibido 23 Abril 2006/Enviado para Modificación 24 Septiembre 2006/Aceptado 26 Octubre 2006

\section{RESUMEN}

Objetivo Analizar el conocimiento y opiniones sobre las políticas de participación en salud y la experiencia con los mecanismos de participación de usuarios, líderes comunitarios y personal de salud en Colombia.

Método Entre 1999 y 2001 se realizó en los municipios Tulúa y Palmira, Colombia, una investigación exploratoria y descriptiva que combinó métodos cuantitativos, encuesta a una muestra de 1497 usuarios, con métodos cualitativos, a través de grupos focales a 210 usuarios y 40 líderes y 36 entrevistas individuales semi-estructuradas a personal de salud. Se realizó un análisis descriptivo de los resultados de la encuesta, mediante el SPSS, y un análisis narrativo de contenido de los datos cualitativos, mediante el Ethnograph.

Resultados La encuesta a los usuarios mostró un gran desconocimiento de la normatividad y los mecanismos de participación formal y un uso limitado de los mismos. Desconocimiento y cierto escepticismo sobre su eficacia se reflejó en el estudio cualitativo con usuarios, líderes y personal de salud. El personal del sector público mostraba un concepto más elaborado y refería una mayor implementación de mecanismos. Entre las dificultades para la implementación destacan carencia de recursos y de cultura participativa.

Conclusiones Los resultados muestran un conocimiento y uso escaso de los mecanismos de participación social en salud, con predominio de los basados en el mercado. Se requiere importante una intervención en información y formación de la población y personal de salud para promover una participación real para el control social del sistema de salud.

Palabras Clave Participación comunitaria, políticas públicas de salud, sistemas nacionales de salud, análisis cualitativo, conocimiento, Colombia, Latinoamérica (fuente: DeCS, BIREME). 


\section{ABSTRACT \\ Knowledge, opinions and experience from applying health participation policy in Colombia}

Objective Analysing knowledge and opinions regarding policies related to social participation in health and how user, community leader and health personnel participation mechanisms are used in Colombia.

Methods An exploratory and descriptive study was carried out between 1999 and 2001 in the towns of Tuluá and Palmira in Colombia using a combination of research methods. A sample of 1,497 health care users were formally surveyed, a focus-group-based qualitative study was made of 210 users and 40 community leaders and 36 semi-structured individual interviews were conducted with health personnel. SPSS was used for a descriptive analysis of survey data and Ethnograph for narrative content analysis of qualitative data.

Results The user survey results revealed a lack of knowledge regarding norms and mechanisms for social participation in health, as well as limited use. Limited knowledge and scepticism regarding its effectiveness was also reflected in the users, leaders and health personnel qualitative study results. Public sector personnel, however, displayed better knowledge and referred to greater implementation of participation mechanisms. Lack of both resources and a culture of participation were amongst the difficulties faced in implementing it.

Conclusions The results indicated scarce knowledge and little use of social participation in health mechanisms (market-based ones predominating). Much greater investment in information and training the population and health system personnel is required as a first step towards promoting real social participation for social control of the health system.

Key Words: Health participation, health policy, health system, qualitative research, knowledge, Colombia, Latin-America (source: MeSH, NLM).

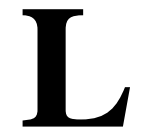

a participación social ha sido incorporada como un componente importante de las políticas de salud dentro de las reformas del sector en numerosos países de todo el mundo $(1,2)$. Sin embargo, no es algo nuevo en Latinoamérica. Desde los años sesenta, aparece articulada a los planes nacionales de desarrollo comunitario, coincidiendo con la efervescencia del desarrollismo (3-4) y defendiendo un modelo contrario a la propuesta de pensadores latinoamericanos, que planteaban una participación autónoma y promovida desde lo local $(5,6)$. Ante las exigencias de los movimientos sociales, en los años setenta se busca un papel más activo de la comunidad en la planificación y ejecución de acciones de interés colectivo, como la salud (7). A finales de la década de los ochenta, 
los países latinoamericanos influidos por un modelo de desarrollo neoliberal, inician reformas del Estado basadas en el mercado. La participación social, que se promueve para la interlocución entre el Estado y la sociedad civil, enfatiza el rol de los usuarios, definidos como "clientes" que pueden participar en el control de la calidad de los servicios (8).

En Colombia, a finales de los ochenta se empieza a implementar una descentralización administrativa y a introducir mecanismos de participación que reciben su impulso definitivo con la Constitución de 1991. La ley 100, que creó el Sistema General de Seguridad Social en Salud (SGSSS) -basado en la competencia entre aseguradoras y prestadores de servicios-, y el decreto 1757(9) promueven la participación como elemento fundamental en la organización y control del sistema. Definen dos tipos de participación en salud: social e institucional en el SGSSS (9). La primera comprende: a) la participación ciudadana para la defensa de los derechos individuales, basada en quejas y reclamos del usuario, a través del Sistema de información y atención al usuario de los servicios de salud (SIAU: línea telefónica permanente, buzón de sugerencias y oficina de atención al usuario, OAU); b) La participación comunitaria, a través de sus organizaciones, en las decisiones de planeación, gestión, evaluación y veeduría. Se establece el Comité para la participación comunitaria (COPACO) como espacio de encuentro municipal entre los diversos actores sociales y el Estado. En segundo lugar, la participación en las instituciones del SGSSS contempla diversos mecanismos: asociaciones de usuarios (AU) para la defensa del asociado y de la calidad del servicio, con un representante en las juntas directivas de proveedores (IPS) y aseguradoras (Empresas Promotoras de Salud, EPS; Administradoras del Régimen Subsidiado, ARS); los comités de ética hospitalaria de las IPS, con dos representantes de las AU y de asociaciones comunitarias de los COPACO. Se define la veeduría -vigilancia de la gestión pública, la prestación de los servicios y la actividad financiera de las instituciones del SGSSS- que se puede realizar a nivel individual por medio de la Dirección Local de Salud; o través de los mecanismos comunitarios e institucionales de participación. En resumen, estas políticas crean numerosos mecanismos para la participación social en salud. Para que la implementación de una política sea efectiva, es necesario que concurran diversos elementos políticos, organizativos y poblacionales (8). Entre los aspectos organizativos, para la participación en salud es fundamental que se creen mecanismos de participación y, además, que la población los conozca (8). Las investigaciones sobre políticas de 
participación social han analizado algunos aspectos relacionados con los actores involucrados -significados de la participación (10-12,17); opiniones sobre las políticas (13); capacidad de las organizaciones comunitarias o instituciones (13-16). Sin embargo, son escasas las investigaciones que determinen el conocimiento de los actores involucrados sobre las políticas, tanto en general (14) como en Colombia (13), donde se ha realizado un importante esfuerzo legislativo. El presente artículo, que presenta resultados parciales de una investigación más amplia $(17,30)$, analiza a) el conocimiento de los usuarios sobre las políticas de participación en salud y su experiencia con los mecanismos de participación; y b) el concepto y opinión del personal de salud sobre la política y su implementación.

\section{METODOLOGÍA}

Área de estudio

El estudio se llevó a cabo en dos municipios del Departamento del Valle del Cauca en Colombia: Tuluá (184 725 habitantes) donde la reforma del sector salud se inició en 1996 y Palmira (283 431 habitantes), cuya reforma comenzó en 1993. Se seleccionaron aplicando los siguientes criterios: haber implementado la reforma del sector salud; poseer población de todos los estratos socioeconómicos; contar con porcentajes altos de afiliación al sistema de seguridad social en salud; disponer de infraestructura en todos los niveles de atención y un municipio con carácter más rural y otro más urbano.

\section{Métodos}

Se realizó un estudio descriptivo, exploratorio, combinando métodos cuantitativos y cualitativos de investigación social. El estudio cuantitativo se basó en una encuesta a usuarios, con un cuestionario estructurado con preguntas abiertas y cerradas sobre aspectos relacionados con la participación en salud, entre otros, el conocimiento de la legislación y de los mecanismos de participación y su utilización. El estudio cualitativo, por su parte, se basó en entrevistas semi-estructuradas individuales (E) y grupos focales (GF), para profundizar en los conceptos y opiniones sobre la participación social en salud y sus mecanismos. Las guías de entrevista (al personal de salud) y grupos focales (a usuarios y líderes) poseían una parte común y otra específica al tipo de entrevistado. Las entrevistas y grupos focales, de 60 a 90 minutos, fueron grabadas y posteriormente transcritas textualmente. El trabajo de campo se realizó entre el 1999 y el 2001. 


\begin{abstract}
Muestra
Para el estudio cuantitativo el tamaño de la muestra se calculó con base en el tamaño de la población del municipio, la tasa esperada de utilización de mecanismos de participación en salud (estimada en $25 \%$ en el estudio piloto), un nivel de confianza del $95 \%$ y una precisión del $3 \%$. Se hizo una selección aleatoria estratificada que incluyera los 6 estratos socioeconómicos. El intervalo de la muestra (cada 67 casas), se calculó sobre el número de domicilios en cada comuna, donde la casa inicial se seleccionó por sorteo. Se procuró entrevistar igual número de hombres y de mujeres para lo cual se hizo muestreo por cuotas. El tamaño final de la muestra fue de 1495 usuarios: 768 en Tuluá (209 hombres y 559 mujeres) y 727 en Palmira (393 hombres y 334 mujeres). En el estudio cualitativo se diseñó una muestra teórica (18), constituida por grupos de informantes que podían influir o verse influidos por la política de participación social en salud, usuarios, líderes comunitarios y distintas categorías de personal de salud: gerentes, coordinadores, encargados de las oficinas de atención al usuario. La muestra de usuarios se seleccionó buscando la variación máxima (19) respecto a edad (18 a 35 años; 36 a 55 años; más de 55); tipo de afiliación al SGSSS (vinculados, subsidiados, contributivos); estrato social (estrato bajo: 1,2; medio: 3,4; alto: 5,6) y sexo. Se entrevistaron 210 usuarios, 130 en Tuluá y 80 en Palmira, en 20 grupos focales y 40 líderes, 18 en Tuluá y 22 en Palmira, en 5 grupos focales. Los líderes eran representantes de ligas de usuarios de las IPS y representantes de la comunidad ante Juntas de Acción Comunal (JAC). El personal de salud se seleccionó teniendo en cuenta la titularidad (pública/privada), el tipo institución (aseguradoras: ARS, EPS; prestadores de servicio de salud: IPS), y categoría laboral (Gerentes y encargado de Oficina de Atención al Usuario). Se entrevistaron 26 gerentes, 13 en Tuluá y 13 en Palmira y 10 encargados de las OAU.
\end{abstract}

Calidad y análisis de los datos

Estudio cuantitativo: En el curso de la encuesta se revisaron todos los cuestionarios y se repitió el $15 \%$ (224) al azar para analizar la consistencia de las respuestas. No se detectaron inconsistencias. Los datos se grabaron en SPSS siguiendo el método de doble entrada y con control automático de inconsistencias. Las respuestas abiertas fueron agrupadas por similitud de contenido semántico y posteriormente codificadas. Se realizó un análisis univariado y bivariado utilizando el programa SPSS WIN 8.0. Se hizo la comparación de proporciones mediante el test de $\mathrm{x}^{2}$ de Pearson y un nivel de significación del 5 \% para el rechazo de la hipótesis nula. 
Estudio cualitativo: Para garantizar la calidad se contrastaron los datos con los entrevistados al terminar la entrevista y se realizó la triangulación de diferentes fuentes de datos, diferentes lugares, diferentes investigadores en la recogida y análisis de datos, diferentes técnicas; además de incluir descripciones amplias. Se hizo un análisis de contenido narrativo con ayuda del programa Ethnograph 5.8. Se elaboraron las categorías de forma mixta a partir de las guías de entrevista y las categorías emergentes de los discursos. Los datos se segmentaron, por municipio, tipo de informante y temas.

\section{RESULTADOS}

Usuarios y líderes

Conocimiento sobre la legislación de participación en salud

De todos los encuestados, sólo el 5,3 \% en Palmira y el 4,1 \% en Tuluá sabía que existe en Colombia alguna disposición legal relacionada con la participación de los usuarios en salud, siendo la Ley 100 la más nombrada, seguida por la Constitución de 1991 y la Tutela. Cuando se les preguntó por sus derechos en salud, el 64,5 \% indicó que es recibir atención médica; el 13,6 $\%$ contar con un trato humanizado por parte del personal de salud y el 7,2 \% tener una atención rápida. Ninguno refirió el derecho a la participación.

En el estudio cualitativo la mayoría de los participantes en los grupos focales, principalmente de Palmira y los mas jóvenes de estrato bajo de Tuluá desconocían las normas relacionadas con la participación de los usuarios en salud y señalaban a las instituciones como responsables de esta situación: "No estamos al tanto de lo que ocurre a nuestro alrededor", "Hace falta comunicación de las instituciones, para uno saber qué se necesita, qué se debe de asistir, cuáles son los derechos que uno tiene" (GF contributivo, 18-35, bajo, Tuluá). Algunas personas de Tuluá, de estrato medio y alto, mencionaron la ley 100, 60 y 10 e indicaron conocerlas a través de sus estudios o ámbito laboral. Por su parte, algunos líderes de ambos municipios mencionan la Ley 100 y la Constitución como normas reguladoras de la participación ciudadana y reconocen que la Constitución les otorga el derecho de participar "La Constitución Política denotaba esos derechos, no recuerdo él articulo para no ir a mentir, pero sé que nosotros tenemos derechos, que participemos como usuarios en cuestiones de salud en beneficio propio de la comunidad". ".......si tenemos el derecho a reclamar en el mejoramiento de la calidad, en la misma ley 100 especifica todo eso, que tenemos 
derecho en caso de que el servicio este mal, reclamar para que se mejore el servicio que esta deficiente" (Líderes Palmira). Algunos líderes manifiestan, no obstante, cierto escepticismo hacia estas normatividades: "Me parece que eso de antemano está en la Constitución Nacional, la participación ciudadana por allá hay unas palabras donde se dice de la veeduría ciudadana, de la participación, pero entonces yo creo que aquí en Colombia es letra muerta", (GF Líderes Palmira)

Conocimiento y experiencia sobre mecanismos de participación

En la encuesta se incluyeron preguntas sobre el conocimiento de los mecanismos de participación, así como su grado de utilización o experiencia. Al preguntársele a los usuarios por cada uno de los mecanismos, afirmaban conocer los mecanismos individuales con más frecuencia que los institucionales (Figura 1). Excepto la OAU, todos los mecanismos eran más conocidos en Palmira, siendo las diferencias estadísticamente significativas ( $\mathrm{p}<0,05$ ), excepto para la línea telefónica (Figura 1). Se hicieron preguntas complementarias para profundizar en el nivel de conocimiento. Si bien la Juntas Directivas era el mecanismo institucional mas conocido (Figura 1), sólo el 7,2 \% lo definía como un mecanismo para el mejoramiento del servicio; un 2.7 \% había participado en la elección de su representante; y un 3,1 \% había recibido información de las juntas por medio de carteleras, volantes o personalmente. En ambos municipios de los pocos que conocían la AU, sólo el 0,9\% lo identificaba como un espacio para el control de la calidad de los servicios. El 1,5 \% de Palmira y el 0,4 \% de Tuluá decía conocer las actividades de los miembros, el 1,7\% había participado alguna vez en reuniones para elegir un representante en la AU, y el 0,1 \% formaba parte de alguna de ellas. En relación a la experiencia con los mecanismos, se observa que la tasa de utilización para todos los mecanismos es de, aproximadamente, 24 \% (Figura 2). En ambos municipios, las mayores tasas de utilización fueron en los mecanismos individuales. Palmira presentó mayores tasas de utilización en todos los mecanismos, con diferencias estadísticamente significativas con Tuluá $(\mathrm{p}<0,05)$ (Figura 2). El estudio cualitativo coincide con lo encontrado en la encuesta. Una constante de la mayoría de los discursos analizados es el escaso conocimiento de los mecanismos de participación en salud contemplados por las normas. Solamente algunos informantes, entre usuarios y líderes, mencionaron algún mecanismo de participación individual, como el buzón de quejas, la OAU y la AU, "si yo he oído que hay unas Oficinas donde lo atienden a uno para quejarse si algo no le gusta a uno..."(G.F contributivo bajo, 18-35 años, Tuluá). "...en cuestión de salud también la gente puede acercase a esa entidad de usuarios para que le solucionen los problemas más graves..."(G.F líderes Palmira). 
En cambio, los entrevistados señalaron diversas estrategias de interacción para lograr una respuesta en salud del Estado o de las instituciones. Por un lado, la utilización de mecanismos individuales formales como la tutela y el derecho de petición para defender los derechos de los individuos, mencionada con mucha frecuencia: " si claro las tutelas, eso esta consagrado en él articulo 23, sería entutelar al Estado para el mejoramiento de la salud, a las EPS o las IPS que este prestando el servicio, para que mire esas falencias que hay y las trate de mejorar" . "Derecho de petición, el derecho de petición es un recurso; que nos facilita el acceso al Estado en estos casos". (Líderes Palmira). Por otro, mencionaban interacciones no formales como la expresión directa de quejas en los servicios o autoridades (ante la superintendencia de Salud; al director de la EPS o IPS, a las autoridades municipales) "hacer llegar oficios o cartas a las autoridades municipales o a las competentes directamente donde se plantee las necesidades de la comunidad" (G.F, contributivo, 36-50 años. Palmira); a través de mecanismos colectivos como organizaciones gremiales "... participar a través del sindicato; poner la queja con el sindicato así sí sería la forma...."(G.F, contributivo, 36-50 años. Tuluá). Muchos entrevistados, sobre todo los jóvenes en Tuluá, indicaban la expresión de reivindicaciones colectivas a través de acciones esporádicas como paros, tomas, asonadas, marchas, para presentar sus quejas en materia de salud: "...con una huelga bien brava" (G.F contributivo, bajo, 18-35 años. Tuluá).

Figura 1. Conocimiento de los mecanismos de participación en salud en Tuluá y Palmira

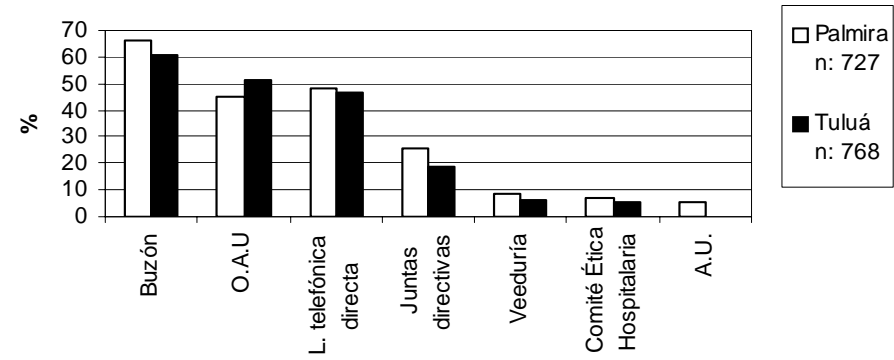

Algunos informantes expresaron que la manera de obtener una respuesta de las instituciones consistía en establecer una interacción clientelista, es decir, recurrir a algún pariente, amigo o líder político con vinculación con el organismo correspondiente, que serviría como "enlace" para poder acceder a éstos: "Conocemos mecanismos como la ayuda o palanca de un familiar o amigo" (G.F contributivo, 18 a 35 bajo Tuluá); "La palanca de un político" (G.F subsidiado y contributivo 36 a 50. Palmira). Finalmente, en el discurso de los informantes, aparecen en relación con los mecanismos de participación expresiones que se 
refieren al uso de los servicios de salud, en sus actividades preventivas o curativas, "brigadas de salud", "campañas de vacunación" "pagar cumplidamente", "asistir a reuniones", "ir a consultas medicas", "hacer buen uso de los servicios de salud".

Figura 2. Tasa de utilización de los mecanismos de participación en salud en Tuluá y Palmira

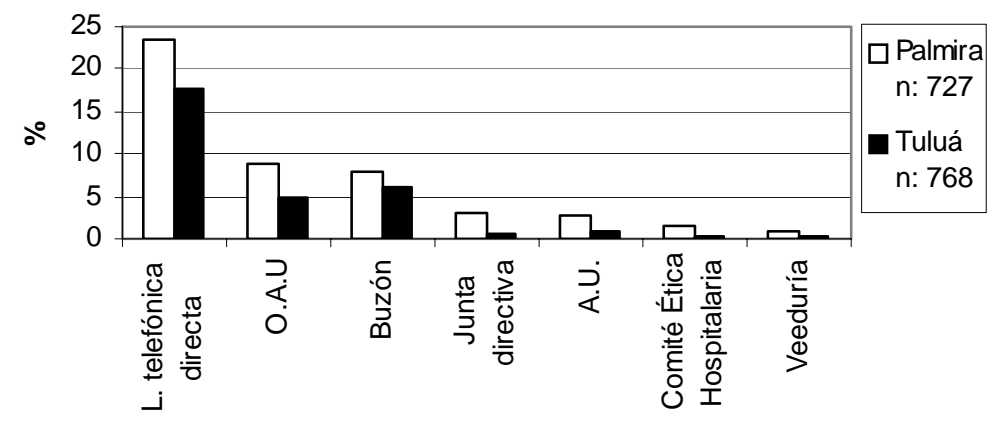

Personal de salud

Conocimiento y opiniones sobre la legislación de participación en salud Entre el personal de salud entrevistado, aunque muchos manifestaron conocer la legislación sobre participación en salud, sólo nombraban la Constitución o la ley 100 como origen de la política pero no ubicaban normas y decretos puntuales. Pocos describieron el artículo 1757, como eje para la participación social que apunta al logro calidad en salud. "La Ley 100 lo establece y obliga con reglamentación de calidad y auditoria". "Se refiere más a lo de calidad en salud el Decreto 1757"(Gerente de IPS pública). En el sector privado se observó un conocimiento más limitado de la normatividad de la participación en salud, que explicitaron directamente, o se hizo evidente por el tipo de respuestas vagas "... la verdad es que si he leído algunas cosas sobre participación y desarrollo comunitario, sobre participación ciudadana, pero que haya profundizado sobre los temas, no" (Gerente EPS privada). Muchos decían que consideraban importante toda la legislación que estructura la participación de los usuarios para darle mayor transparencia y calidad a la prestación de los servicios. No obstante, también expresaban bastante escepticismo en cuanto a su eficacia e identifican diversas causas, como la falta de recursos técnicos y financieros para implementarla, la saturación de normas en el país, la falta de claridad de la norma: "....simplemente es un Decreto más, una Ley más, buena si, pero eso está es en papel, la aplicabilidad no está, es difícil, a la gente se la deja sola, entonces no es sacar un decreto y ya... yo creo que estamos en un país donde todo son decretos, pero pues lo real es que no existe nada cierto en normas y leyes, tenemos más de dos millones quinientos mil normas y decretos y todo ese tipo de cosas y de eso ¿qué se aplica?, o sea, la funcionalidad de esos decretos 
¿dónde está?. No existe, si usted va a buscar algún recurso no lo encuentra. Son más los esfuerzos, los sacrificios, lo que hay que hacer para sacar un peso, para obtener un recurso que el trabajo en si, o sea es un poco complicada la situación". (Gerente de IPS pública). ".son útiles en el papel pero en la realidad no... no hay forma, ni dinero para poner a funcionar toda esa normatividad" (Gerente EPS pública) "...no creo..la Ley tiene mucho hueco, mucho vacío en la prestación del servicio, es inconsistente en algunas cosas...no esta claramente definido como va a participar el usuario, por ejemplo en el comité técnico científico, no existen procedimientos claros para su participación" (Gerente EPS Privada). Otros identificaban las dificultades para implementarla en factores inherentes a los usuarios como su falta de conocimiento, de motivación, de competencias adecuadas y de tiempo "...yo opino que esa Ley, pues esta muy bien escrita en el papel, pero pues no creo que se lleve mucho a la práctica, más no porque las E.P.S. no quieran, sino porque los mismos usuarios o no tienen el tiempo o no les interesa, o no pueden o no ven la importancia de esto..." (Gerente EPS-ARS privada), "....no ceo que funcione, porque el usuario no tiene tiempo para participar, y además tendría que manejar muy bien la ley...."(Gerente EPS Privada).

Conocimiento y opinión sobre los mecanismos de participación

En los testimonios de los entrevistados se observó, que el personal de salud de las instituciones públicas conocía mejor los mecanismos de participación, pero sobre todo los mecanismos de participación institucionales como las AU, comités de veeduría, o juntas directivas hospitalarias. Además, algunos informantes de EPS públicas, señalaron el Derecho de Petición y la Tutela como un mecanismo efectivo para obtener respuesta del sistema de salud "...conozco la Asociación de usuarios...existen buzones de sugerencias y reclamos, existe la línea telefónica...La acción de Tutela ha sido un mecanismo que me parece bueno en el sentido de que la gente ya sabe que si la queja o derecho de petición no funcionó, la tutela al otro día ya esta operando su respuesta." (Gerente EPS pública). El personal de las instituciones privadas demostró conocer menos los mecanismos formales de participación en salud, o mencionaban casi siempre mecanismos individuales como la OAU, la línea telefónica y el buzón de quejas, y algunas veces nombraron las AU o las juntas administradoras, como espacio de participación "...conocer los mecanismos como tal, no, pero existe la asesora del servicio al cliente, que está dedicada las 8 horas, a atender y resolver todo este tipo de cosas." (Gerente IPS privada). Otros señalaron como formas de participación la información a la comunidad a través canales de comunicación informales, como los líderes o visitas a la comunidad "No tengo conocimiento de cuales son los mecanismos de participación de los usuarios en los servicios de salud, pero de todas maneras nosotros tenemos mucho contacto con los líderes....se les informa acerca de programas y planes" (Gerente IPS privada). ".... las visitas que se les hace a los usuarios en sus casas para que nos digan como les parece el servicio...."(Coordinadora de Servicios EPS privada). 
160 REVISTA DE SALUD PÚBLICA · Volumen 8 (3), Noviembre 2006

Algunos opinan que los mecanismos son necesarios para mejorar la calidad de los servicios "..... es bueno, es lo mismo de antes, eso ayuda a mejorar porque si uno no conoce qué es lo que pasa y qué es lo que piensa la gente del servicio, poco se puede hacer para mejorar" (Coordinadora IPS Mixta). En cambio, muchos opinaron que no funcionan por diversos motivos, entre ellos la falta de un anclaje cultural de la participación en los usuarios y en las instituciones "es importante, pero estamos conscientes de que hace falta recorrer bastante camino para que en realidad la comunidad y el usuario se integren a las decisiones administrativas, yo pienso que en este momento la participación en esa parte es bastante deficiente." (Gerente IPS Pública). "No creo que esto funcione todavía, primero hay que darles todas las herramientas necesarias para que entiendan con que fin se hizo..." (EPS privada). Varios informantes del sector público, consideran que los mecanismos no funcionan por falta de recursos técnicos y financieros: "las veedurías ciudadanas, comités de participación comunitaria, asociaciones y ligas de usuarios en el sector salud, pero no funcionan idóneamente o como quisiéramos, porque no hay como..." (Gerente IPS Pública). Otros señalan que los mecanismos no han logrado los objetivos para los que fueron creados, convirtiéndose, en estrategias para hacer reclamos o presentar quejas, para revisar y solucionar casos particulares, pero sin lograr cambios estructurales en la prestación de los servicios: "En lo que se llama liga de usuarios, yo lo veo como un espacio de expresión de quejas y son quejas que desde mi punto de vista no generan transformación, generan respuestas o cambios frente a ese problema, pero es el problema de esa persona y no de la organización frente a otras personas". (Gerente de IPS pública). Algunos informantes consideran que es mejor recurrir a los mecanismos como las encuestas: "Una liga de usuarios, no es necesario para que la empresa sea excelente, existen otros mecanismos, como las encuestas, las llamadas etc., existen empresas excelentes que los usan con muy buenos resultados" (Gerente EPS Privada).

Mecanismos de participación implementados

De acuerdo a los testimonios de los entrevistados, las instituciones del sector público han implementado mayor número de mecanismos de participación individuales y comunitarios. Todas las instituciones públicas decían tener AU, algunas comités de veeduría, y solo una tenía un representante de la AU a la junta directiva: “....tenemos un departamento de atención al usuario, que es el que se encarga en la parte administrativa de orientar a cada uno de los usuarios, sobre sus características como usuario del hospital y también sobre cuáles son sus derechos y cuales son las formas que tienen para acceder a los servicios del hospital. Existe también una asociación de usuarios que tiene un representante en la Junta Directiva del Hospital". (Gerente IPS Pública). Los gerentes de las ARS e IPS mixtas entrevistados reportaron algunos mecanismos de participación implementados como AU y representantes en las Juntas directivas: “..... tenemos Liga de usuarios con 7 personas. Junta directiva donde participan los usuarios asociados. Buzón de 
quejas y reclamos. No lo han colocado de nuevo porque se lo llevan!. (Gerente ARS Mixta). Los informantes del sector privado indicaron haber implementado sobre todo mecanismos de participación individual, y pocas veces AU.

Algunas instituciones, sobre todo las privadas, se limitan a conocer la opinión sobre el servicio mediante mecanismos como encuestas, entrevistas y comunicación directa entre usuarios e instituciones: "nosotros tenemos 4 tipos de encuestas para los pacientes, tenemos visita directa diariamente a los pacientes, para que nos estén contando sobre su satisfacción, son 4 formularios diferentes, uno para urgencia, uno para consulta externa y otros para especialización y el seguimiento que le hacemos a las historias clínicas". (Gerente IPS privada Tuluá). "Existe una línea telefónica 9800, que comunica directamente con la Presidencia del Seguro a nivel nacional" (Gerente de EPS pública).

Casi todos los actores institucionales opinaban que había sido muy difícil la implantación de los mecanismos, y lo atribuyen a factores relativos a la población como su falta de compromiso, de conocimiento, de sentido de pertenencia, actitudes y creencias que obstaculizan su participación: "Se citó a una reunión a los integrantes de la asociación de usuarios para el 25 de enero a la que solo asistió una sola persona. A veces es frustrante es, porque es como haber preparado todo un trabajo en donde se iba a hacer un taller que iba a brindar muchas cosas, muchas inquietudes de la comunidad para la IPS...." (Coordinadora IPS Privada.). "Es un trabajo muy difícil, porque hay que darles a conocer la importancia de participar y hacerles entender que no es perder el tiempo" (Coordinadora EPS-ARS privada. Tulúa) «Yo creo que es la falta de participación de los mismos usuarios porque ellos no conocen para que es la AU...."(Gerente IPS mixta).

Otros entrevistados consideran que la dificultad en la implantación de los mecanismos de participación se originan en factores institucionales, como la falta de recursos técnicos y financieros; las actitudes gerenciales con una falta de voluntad para implementarlos; a falta de capacitación de los actores institucionales "En primer lugar decisiones y gestión administrativas, yo creo que aquí en la parte gerencial ha existido conciencia pero no la suficiente voluntad para vincular a la comunidad en la participación y la prestación de servicios (Gerente IPS Pública). "Disposición de recursos para los sistemas de información... la falta de divulgación por asignación de recursos. La reglamentación está clara, pero las instituciones, la mayoría de las veces hacen caso omiso de estas normas y no las cumplen, por problemas de recursos o de actitudes gerenciales. Que el Ministerio de Salud no es muy claro, en el sentido que exige unos mecanismos que a veces son imposibles de cumplir porque su aplicación no es viable por x o y razón, la institución no trabaja así y el Ministerio no vigila el cumplimiento de dicha obligación" (Gerente IPS pública). 


\section{DISCUSIÓN}

Un resultado destacable de este estudio es el conocimiento limitado acerca de las normas sobre participación social en salud, por parte de los usuarios, líderes y de la mayoría del personal de salud entrevistado. Resultados semejantes fueron obtenidos por un estudio realizado poco antes en otra área de Colombia, que mostraba un escaso conocimiento de la política y del nuevo sistema de salud por parte de los usuarios (13). El personal de salud entrevistado no parece poseer un conocimiento orgánico, es decir, sistemático, global y organizado acerca de la legislación en participación. Aunque los informantes del sector público manifestaron en su mayoría conocer la legislación, con algunas excepciones, no lograban hacer una descripción detallada de sus contenidos. El desconocimiento de la legislación por parte del personal del sector privado era mayor que en el sector público, lo cual podría indicar la percepción de muchos de estos actores, de que la política de participación social es una estrategia de control a los servicios de salud que debe cumplirse sobre todo en el sector público $(11,17)$. En este estudio sólo se ha medido a través de la encuesta el nivel de conocimiento de los usuarios; no obstante, la correspondencia entre estos resultados y los grupos focales a usuarios (19), así como el haber alcanzado la saturación de la información en el estudio cualitativo (18), señalan hacia la validez de los resultados y permite suponer que una encuesta entre los otros grupos de informantes arrojaría resultados similares.

Muchos líderes y personal de salud mostraban una opinión favorable hacia la promoción de la participación de los usuarios en el control de la calidad de los servicios de salud, pero no dejaban de manifestar su escepticismo. Entre las causas identificaban diversos factores institucionales como falta de claridad de la norma, inexistencia de recursos técnicos y financieros y la saturación de normas en el país, que determinan las posibilidades de implementación y, además, podrían contribuir a agotar el comportamiento participativo de las comunidades y ciudadanos. Estos aspectos indican limitaciones en el propio diseño de la política de participación, ya que para poder ser considerada como tal, debería ir acompañada de los recursos necesarios para su implementación (20). Por otro lado, varios autores $(21,22)$ argumentan que una gran cantidad de llamados institucionales a participar fragmenta lo local de lo global, lo sectorial de lo territorial, lo público de lo privado, porque muchas propuestas se ubican en espacios sociales y políticos divididos, sin aparente relación entre sí, fragmentando en lugar de articular el comportamiento participativo, como persiguen las políticas. 
Los usuarios poseen un mejor conocimiento de los mecanismos de participación de carácter individual, como el buzón de quejas, OAU y Línea telefónica. Podría reflejar el hecho que estos mecanismos han sido más implantados en las instituciones, tanto públicas como privadas y por tanto son fácilmente identificables por ser tangibles y observables. También podría estar influido por el hecho de ser estrategias de mercado utilizadas en establecimientos frecuentados cotidianamente, como los supermercados o establecimientos diversos de venta de bienes o servicios. La línea telefónica se utiliza también para aspectos diferentes a la expresión de opiniones o intercambio de informaciones, sobre todo a la atención (citas, ambulancia, etc.). Resultados similares se encontraron en otros estudios en Colombia (13) y en Brasil (14). Igualmente, es importante señalar que la gran mayoría de usuarios y muchos líderes se refieren a la Tutela y el Derecho de Petición como mecanismo para obtener respuestas efectivas del Estado y de las instituciones de salud. Es una forma de intermediación entre el Estado y la sociedad civil, creada por la constitución del 1991 para defender los derechos ciudadanos, muy utilizada. Ya en el año 1998 el Ministerio de Salud presentó ante el Congreso del país un informe (23), donde planteaba su preocupación por la gran cantidad de tutelas de los usuarios y por tanto, el aumento presupuestal al que tenían que recurrir para responder a esas demandas. Según estudios recientes de la Defensoría del Pueblo, más del 3\% de las tutelas interpuestas en el país obedecen al ámbito de la protección del derecho a la salud y se falla a favor del tutelante en un 78,5 \% de los casos (24). Parecería que, por la difusión que se hace en los medios masivos de comunicación o por otras vías, se han ido conformando imaginarios colectivos acerca de la efectividad de esa estrategia, pero fundamentalmente para obtener respuestas a problemas individuales de atención. En contraste, los resultados muestran un muy escaso conocimiento de los usuarios sobre los mecanismos colectivos para la participación en las instituciones del SGSSS que estaría revelando el exiguo fortalecimiento y desarrollo de una cultura participativa, en la cual deberían estar mas comprometidas las instituciones y el Estado, considerando que se trata de los mecanismos que conducen a la toma de decisiones, tal como promueven las políticas. La escasa difusión del conocimiento sobre la política de participación se podría explicar a partir de múltiples factores, pero parece indudable que la ausencia de una estrategia educativa e informativa hacia la comunidad y el propio personal es determinante. Para que la participación se produzca de una manera eficiente y democrática en ésta nueva relación sociedad civil- Estado, es fundamental el acceso libre y democrático a la información (25). 
En el contexto de la democracia representativa, que rigió por muchos años la relación Estado-sociedad civil en Colombia, se contaba con estrategias tradicionales de mediación como la representación partidista y gremial y su desvirtuación, en la que los agentes políticos establecían relaciones de “clientela” con sus representados (25). Estas prácticas clientelistas emergen en los testimonios de muchos líderes y usuarios como canales efectivos para interactuar con el Estado o las instituciones. Las prácticas “clientelistas” son propias de una sociedad que no cuenta con suficientes organizaciones sociales y comunitarias críticas, propositivas e independientes de la manipulación y sometimiento a los políticos (26). Este tipo de relaciones, y la obvia politización y cooptación que hacen algunos líderes, disminuye las posibilidades de una participación autónoma y permanente (27) y se constituye en una barrera importante para la participación en salud (13). La frecuente mención por parte de usuarios, líderes y aun de algunos del personal de salud acerca de la tutela, derechos de petición, presentación directa de quejas a autoridades, acciones clientelistas y formas de hecho o para-legales, como estrategias de mediación entre el Estado- las instituciones de salud y ellos, podría reflejar por un lado falta de información acerca de mecanismos institucionales de participación; pero también, parece mostrar una falta de respuestas oportunas y satisfactorias por parte de las instituciones o del Estado.

A excepción de la línea telefónica, las tasas de utilización de los mecanismos individuales estuvieron por debajo del $9 \%$ y de los mecanismos de participación colectiva, por debajo del 3 \%. Estas tasas de utilización de los mecanismos de participación, tanto individuales como colectivos, fue similar a las tasas encontradas en los estudios de Colombia (13) y Brasil (14). No obstante, las investigaciones en este sentido son escasas, por lo que no se puede establecer cuales son las tasas adecuadas en el uso de los mecanismos para el control social de la salud y para qué tipo de mecanismos. Los datos proporcionados por el estudio, tanto en relación a conocimientos, como utilización, proporcionan unos resultados de referencia que permitirán analizar en el futuro cambios en relación a la efectividad de la implantación de las políticas de participación.

La población rural de Palmira presentó un mayor conocimiento de los mecanismos de participación y una mayor tasa de utilización de estos, diferencia significativa estadísticamente con la población urbana de Tuluá. Aunque se necesitaría un mayor análisis, se podría relacionar con el amplio universo de tradición participativa de los sectores campesinos en el país, desde 
la cooperación y solidaridad de profunda raigambre ancestral, hasta organizaciones y asociaciones campesinas con las cuales se han incorporado o han respondido a los distintos momentos de modernización del agro Colombiano (21). Aunque el movimiento campesino se encuentra actualmente en crisis entre otras cosas por la guerra, ha sido un protagonista importante en la vida nacional, lo cual deja profundas raíces en la memoria colectiva y en las prácticas sociales de trabajo comunitario.

$\mathrm{Al}$ igual que con el conocimiento, es en el sector público donde se refiere una mayor implantación de los mecanismos de participación, situación igualmente observada en otra área de Colombia (13). Probablemente se debe a que, en las instituciones públicas y mixtas se siguen las normas, mientras que se exime a las instituciones privadas de aplicar varias de carácter colectivo para la toma de decisiones, y a la falta de capacidad institucional del ente regulador para hacer cumplir la norma en el sector privado (13) situación particularmente común en los países de renta media o baja (28).

En conclusión, las políticas de participación social impulsadas por las reformas del sistema de salud en Colombia parecen haber generado algunos cambios relacionados con la expresión de quejas, es decir, aquellos que reflejan una interacción entre la población y los servicios basada en relaciones de mercado. En cambio, el conocimiento e implantación de mecanismos relacionados con la toma de decisiones colectivas es escaso. Estos mecanismos, así como su difusión y fortalecimiento, deberían constituir el núcleo central si se persigue, como manifiestan las políticas, una participación social como forma de interacción democrática y directa, es decir, una nueva relación de la población y el Estado, para una efectiva construcción conjunta de lo público. Sólo así, se generaría una efectiva planeación, ejecución, vigilancia y control de la gestión del servicio público de salud, propósito fundamental de la política. Parece ser que la norma no ha podido contribuir a crear una cultura participativa en salud; o como plantea García Canclini (29), a partir de la desaparición de las funciones del Estado, o el traspaso de éstas a corporaciones privadas, se va creando una cultura participativa organizada a través del consumo, mas que del ejercicio ciudadano.

Agradecimientos. Estos resultados son parte de la investigación realizada en asociación con el Institute for Health Sector Development (Inglaterra), GEMA-Universidad del Valle (Colombia), Universidad Federal de Pernambuco y el Instituto Materno Infantil de Pernambuco (IMIP, Brasil), Escuela Andaluza de Salud Pública (España), con el 
apoyo del Consorci Hospitalari de Catalunya y las Secretarías de Salud de los municipios de Tuluá y Palmira, y la Secretaría de Salud del Departamento del Valle del Cauca, en Colombia. Agradecemos a las personas entrevistadas en estos dos municipios que aceptaron compartir con los investigadores sus opiniones, ideas e inquietudes y a la Comisión Europea DG XII por su ayuda financiera (Contrato: IC18-CT98-0340).

\section{REFERENCIAS}

1. Broomberg J. Managing the health care market in developing countries: prospects and problems. Health policy and planning 1994; 9(3): 237-251.

2. Isaza P. La reforma del sector salud. Educ Med Salud 1995; 29(3): 270-285.

3. Ugalde A. Ideological dimensions of community participation in Latin America health programes. Soc. Sci.Med 1985; 21:41-53.

4. Carranza M y Almeida E. La Psicología Comunitaria en México. En: Almeida, E. Martínez M y Varela M. Psicología Social Comunitaria. Facultad de Psicología de la Universidad Autónoma de Puebla, México 1995; Número Especial (1):13-130

5. Freire P. La Educación como práctica de la Libertad. México: Siglo XXI; 1984.

6. Fals Borda O. Conocimiento y poder popular. Bogotá: Siglo XXI y Punta de Lanza; 1984.

7. OMS- UNICEF. Atención primaria en Salud. Alma Ata 1978. Salud para todos. Serie \# 1.Génova: OMS; 1978

8. Vázquez ML, Siquiera E, Kruze I, Da Silva A, Leite IC. Los procesos de reforma y la participación social en salud en América Latina. Gaceta Sanitaria. 2002; 16(1): 30-38.

9. Ministerio de Salud de Colombia, Decreto 1757. Por el cual se organizan y establecen las modalidades y formas de participación social en la prestación de los servicios de salud. Bogotá, Colombia; 1994.

10. Vázquez ML, Ferreira MR, Siqueira E, Pereira AP, Diniz A, Leite I et al. Visión de los diferentes agentes Sociales sobre la participación social en el sistema de salud en el Nordeste de Brasil. Una Aproximación Cualitativa. Rev Esp de Salud Pública 2002; 76(5): 585-594.

11. Vázquez ML, Ferreira MR, Siqueira E, Kruze I, Diniz A, Veras I et al . Participação social nos serviços de saúde: concepções dos usuários e líderes comunitários em dois municípios do Nordeste do Brasil. Cad de Saúde Pública, Rió de Janeiro 2003; 19 (2): 109-118.

12. Bosi ML, Affonso K. Cidadania, participação popular e saúde: com a palabra, os usuários da Rede Pública de Serviços. Cad Sáude Pública 1998; 14 (Sup 2): 59- 68.

13. Mosquera M, Zapata Y, Lee K, Arango C, Varela A. Strengthening user participation through health sector reform in Colombia: a study of institutional change and social representation. Health Policy and Planning 2001; 16(2):52-60. 
14. Vázquez ML, Ferreira MR, Siqueira E, Diniz A, Pereira AP, Veras I et al. Nível de informação da população e utilização dos mecanismos institucionais de participação social em saúde em dois municípios do Nordeste do Brasil. Ciência \& Saúde Coletiva 2005; 10(suppl.): 141-155.

15. Arévalo DA. Participación Comunitaria y control social en el sistema de salud. Rev Salud pública Bogotá 2004; 6(2):107-139.

16. Abrantes-Pêgo A. Participación social en salud: un estudio de caso en Brasil. Salud Pública de México 1999; 41 (6): 466-474.

17. Delgado ME, Vázquez ML, Zapata Y, Hernán, M. Participación social en salud: conceptos de usuarios, líderes comunitarios, gestores y formuladores de políticas en Colombia. Una mirada cualitativa. Rev Española de Salud Pública 2005; 79 (6):697-707.

18. Fernández de Sanmamed, MJ. Diseños de estudios y diseños maestrales en investigación cualitativa. En: Vázquez ML, Ferreira MR, Mogollón AS, Fernández de Sanmamed MJ, Delgado ME, Vargas I. Introducción a las técnicas cualitativas de investigación aplicadas en salud. Barcelona: Serveis de Publicacions UAB 2006.

19. Patton Q. Qualitative evaluation and research methods. London: Sage; 1990.

20. Walt G. Health policy. An introduction to process and power. London: Zed Books. 1994.

21. Vargas A. Participación social. Una mirada critica. Bogotá: Almudena; 2000.

22. Vega JE. Ideal democrático y democracias real en América Latina. En Dos Santos M. (Coordinador). Que queda de la representación política?. Caracas: Nueva sociedad-CLACSO; 1992.

23. Ministerio de Salud de Colombia. Informe de Gestión. Bogotá; 1998.

24. Defensoría del Pueblo. La tutela y el derecho a la salud. Causas de las tutelas en salud. Bogotá Colombia; 2005.

25. Restrepo D. Participación social: Relaciones Estado- sociedad civil. Rev de Salud Pública 2001; 3 (3): 130-143.

26. Santana P. Los movimientos sociales en Colombia. Bogotá: Ediciones Foro Nacional por Colombia; 1989.

27. Delgado M.E, Carranza M. Perspectiva Psicosocial de los Movimientos Sociales. Escuela de Psicología UAS, México. Revista Psicólogos. 1993; (1):1825.

28. Kumaranayake L. The role of regulation: influencing private sector activity within health sector reform. J. Int. Develop.. 1997; 9(4):641-649.

29. García NC. Consumidores y ciudadanos. México: Grijalbo; 1995.

30. Delgado ME, Vázquez ML. Barreras y oportunidades para la participación social en salud en Colombia: percepciones de los actores principales. Rev de Salud Pública 2006; 8(2):128-140. 